\title{
The Art of Extracting One-Dimensional Flow Properties from Multi-Dimensional Data Sets
}

\author{
R. A. Baurle* and R. L. Gaffney* \\ NASA Langley Research Center, Hampton, Va 23681
}

\begin{abstract}
The engineering design and analysis of air-breathing propulsion systems relies heavily on zero- or one-dimensional properties (e.g. thrust, total pressure recovery, mixing and combustion efficiency, etc.) for figures of merit. The extraction of these parameters from experimental data sets and/or multi-dimensional computational data sets is therefore an important aspect of the design process. A variety of methods exist for extracting performance measures from multi-dimensional data sets. Some of the information contained in the multi-dimensional flow is inevitably lost when any one-dimensionalization technique is applied. Hence, the unique assumptions associated with a given approach may result in one-dimensional properties that are significantly different than those extracted using alternative approaches. The purpose of this effort is to examine some of the more popular methods used for the extraction of performance measures from multi-dimensional data sets, reveal the strengths and weaknesses of each approach, and highlight various numerical issues that result when mapping data from a multi-dimensional space to a space of one dimension.
\end{abstract}

\section{Introduction}

The design and analysis of high-speed air-breathing propulsion systems has historically relied heavily on modular ${ }^{1,2}$ or quasi-one-dimensional ${ }^{3}$ cycle analysis codes for performance assessment. Examples from this class of tools include the RamJet Performance Analysis (RJPA) ${ }^{4}$ code developed at the Applied Physics Labs, the GASL1D ${ }^{5}$ code developed at the General Applied Science Labs, and the SRGULL ${ }^{6}$ code developed at the NASA Langley Research Center. The high-end parallel computing capabilities that exist today, coupled with the maturation of algorithms for the integration of partial differential equations, have significantly reduced the turn-around time required for high-fidelity Reynolds-Averaged Navier-Stokes simulations. These advances, accompanied by the parallel development of advanced grid generation and flow visualization tools, have allowed the use of multi-dimensional analysis for a variety of scramjet engine components. The current state-of-the-art processes for high-speed propulsion component design and analysis involves a combination of one-dimensional and multi-dimensional analysis approaches. This scenario necessitates the need to reduce (or increase) the dimensionality of the analysis results where an exchange of data between the various approaches is required. The reliance on multi-dimensional approaches in the design and analysis of engine components will inevitably increase as high-performance computing capabilities continue to improve. The need to extract performance estimates, however, will still require an ability to relate the multi-dimensional flowfield to an equivalent one-dimensionalized representation.

This paper compares a variety of one-dimensionalization techniques commonly employed for internal flows. The various strengths and weaknesses of each approach are highlighted for a variety of flowfields ranging from purely analytical solutions to realistic scramjet flowpaths. The goal of this paper is not to suggest that one procedure be employed in lieu of another. Instead, the various characteristics of each approach are presented with detailed discussions describing why significant differences often appear between one-dimensionalized properties obtained from different methodologies. Suggestions for dealing with key numerical issues are also discussed, when appropriate.

\footnotetext{
*Aerospace Engineer, Hypersonic Airbreathing Propulsion Branch, Senior Member AIAA.

Copyright (c) 2007 by the American Institute of Aeronautics and Astronautics, Inc. The U.S. Government has a royalty-free license to exercise all rights under the copyright claimed herein for Governmental purposes. All other rights are reserved by the copyright owner.
} 


\section{One-Dimensionalization Techniques}

In general, one-dimensionalization techniques can be categorized as either a weighted or flux-based approach. The weighted approaches are easy to implement, tend to yield uniform properties that "visually" mimic the original multi-dimensional data, and tend to maintain the qualitative physical features of the parent multi-dimensional flow (e.g. non-decreasing entropy changes). The dilemma with these approaches is that fluxes reconstructed from the weighted variables will, in general, not match those obtained from the multi-dimensional data set. As a result, this averaging approach is not well suited for coupling a multidimensional analysis with one-dimensional engineering analysis tools. Flux-based approaches attempt to address this deficiency by formulating a set of one-dimensional flow properties that precisely reproduce some specified set of fluxes from the multi-dimensional data set. Three different flux-based approaches are considered in this effort: the Conserved Mass/Momentum/Energy (CMME) method (sometimes referred to as the stream-thrust average), the Conserved Mass/Momentum/Energy approach with the Langley distortion methodology, ${ }^{7}$ and the Conserved Mass/Energy/Entropy (CMES) method (referred to as the thermodynamic state average in Refs. 8,9). The mapping of the multi-dimensional flowfield to a one-dimensional representation is realized by applying a given one-dimensionalization approach to a family of computational surfaces (or lines in two dimensions) as illustrated in Fig. 1. The surfaces of interest will generally correspond to the cross-flow planes.

\section{Weighted Average}

Weighted approaches are generally expressed as,

$$
\phi=\frac{\int \phi \mathrm{w} d A}{\int \mathrm{w} d A}
$$

where $\phi$ is the property to be one-dimensionalized, w is the weighting function, and $A$ is the area over which the average is being performed. Popular choices for the weighting factor are $\mathrm{w}=1$ (area-weighting) and $\mathrm{w}=\rho(\overrightarrow{\mathrm{v}} \cdot \vec{n})$ (mass flux-weighting). Other weighted approaches, particularly those designed for experimental data sets that are often incomplete, are discussed elsewhere. ${ }^{10,11}$

\section{Conserved Mass/Momentum/Energy Method}

The CMME method produces a set of uniform flow properties that satisfy the integral relations for conservation of mass, momentum, and energy, i.e.,

$$
\begin{aligned}
f_{\text {mass }}^{m} & =\int\left[\rho(\overrightarrow{\mathrm{v}} \cdot \vec{n}) Y_{m}\right] d A \\
\vec{f}_{\text {momentum }} & =\int[\rho(\overrightarrow{\mathrm{v}} \cdot \vec{n}) \overrightarrow{\mathrm{v}}+P \vec{n}] d A \\
f_{\text {energy }} & =\int[\rho(\overrightarrow{\mathrm{v}} \cdot \vec{n}) H] d A
\end{aligned}
$$

where $f$ represents the flux quantities being conserved, $\rho$ is the mixture density, $\overrightarrow{\mathrm{v}}$ is the velocity vector, $\vec{n}$ is the unit vector normal to the surface of integration, $Y_{m}$ is the mass fraction of species " $m$ ", $P$ is the static pressure, and $H$ is the total enthalpy (sum of the static enthalpy and kinetic energy).

The uniform flow properties that satisfy these integral flux relations are defined based on the following expressions,

$$
\begin{aligned}
f_{\text {mass }}^{m} & =\left[\boldsymbol{\rho}(\overrightarrow{\mathbf{v}} \cdot \overrightarrow{\mathbf{n}}) \mathbf{Y}_{\mathbf{m}}\right] \mathbf{A} \\
\vec{f}_{\text {momentum }} & =[\boldsymbol{\rho}(\overrightarrow{\mathbf{v}} \cdot \overrightarrow{\mathbf{n}}) \overrightarrow{\mathbf{v}}+\mathbf{P} \overrightarrow{\mathbf{n}}] \mathbf{A} \\
f_{\text {energy }} & =[\boldsymbol{\rho}(\overrightarrow{\mathbf{v}} \cdot \overrightarrow{\mathbf{n}}) \mathbf{H}] \mathbf{A}
\end{aligned}
$$

where the bold-faced quantities denote one-dimensional parameters. The equation set is closed by introducing an equation of state, e.g. $\mathbf{P}=\boldsymbol{\rho} \mathbf{R T}$. This method results in a nonlinear system of coupled equations, and the procedure used to decode the one-dimensional flow properties from this equation set is given in Appendix A. 
It should be noted that the properties extracted from this methodology result in an effective uniform flow characterization of the multi-dimensional flowfield, and is not necessarily a one-dimensionalization of the flowfield (i.e. cross flow velocity components may be present). If one-dimensional properties are desired, then one should convert the momentum vector into a scalar momentum equation (i.e. a stream-thrust equation). This step is accomplished by defining the unit normal, $\overrightarrow{\mathbf{n}}$, to be parallel to the "streamwise" direction, and taking the dot product of this vector with the momentum vector prior to the decomposition process.

\section{Langley Distortion Methodology}

The Langley distortion methodology ${ }^{7}$ satisfies the same flux expressions given in Eq. 2, but additional flux relations are introduced to provide information on the impact of three-dimensional effects (i.e. flow distortion). The additional flux relations are the mass flux-weighted kinetic energy components, $\int \rho(\overrightarrow{\mathrm{v}}$. $\vec{n}) u^{2} d A, \int \rho(\overrightarrow{\mathrm{v}} \cdot \vec{n}) v^{2} d A, \int \rho(\overrightarrow{\mathrm{v}} \cdot \vec{n}) w^{2} d A$, and the pressure force components, $\int P \vec{n} d A$. This additional information simplifies the decomposition process, since the velocity (via the kinetic energy components) and the pressure are readily available, but results in an over-constrained system of equations. Additional unknowns (distortion parameters) are introduced to allow the uniform flow properties to simultaneously satisfy these constraints and the desired conservation relationships (Eq. 2). The uniform flow properties obtained from the Langley distortion methodology satisfy the following expressions,

$$
\begin{aligned}
f_{\text {mass }}^{m} & =\left[\boldsymbol{\rho}(\overrightarrow{\mathbf{v}} \cdot \overrightarrow{\mathbf{n}}) \eta_{1} \mathbf{Y}_{\mathbf{m}}\right] \mathbf{A} \\
f_{\text {momentum }} & =\left[\boldsymbol{\rho}(\overrightarrow{\mathbf{v}} \cdot \overrightarrow{\mathbf{n}}) \eta_{2}(\overrightarrow{\mathbf{v}} \cdot \overrightarrow{\mathbf{n}})+\eta_{4} \mathbf{P}_{\mathrm{ref}}\right] \mathbf{A} \\
f_{\text {energy }} & =\left[\boldsymbol{\rho}(\overrightarrow{\mathbf{v}} \cdot \overrightarrow{\mathbf{n}})\left(\mathbf{h}+\eta_{3}(\overrightarrow{\mathbf{v}} \cdot \overrightarrow{\mathbf{n}})^{2} / 2\right)\right] \mathbf{A}
\end{aligned}
$$

where

$$
\begin{aligned}
\eta_{1} & =\frac{\int \rho(\overrightarrow{\mathrm{v}} \cdot \vec{n}) d A}{\boldsymbol{\rho}(\overrightarrow{\mathbf{v}} \cdot \overrightarrow{\mathbf{n}}) \mathbf{A}} \\
\eta_{2} & =\frac{\int \rho(\overrightarrow{\mathrm{v}} \cdot \vec{n})(\overrightarrow{\mathrm{v}} \cdot \overrightarrow{\mathbf{n}}) d A}{\boldsymbol{\rho}(\overrightarrow{\mathbf{v}} \cdot \overrightarrow{\mathbf{n}})(\overrightarrow{\mathbf{v}} \cdot \overrightarrow{\mathbf{n}}) \mathbf{A}} \\
\eta_{3} & =\frac{(\overrightarrow{\mathbf{v}} \cdot \overrightarrow{\mathbf{v}})^{2}}{(\overrightarrow{\mathbf{v}} \cdot \overrightarrow{\mathbf{n}})^{2}} \\
\eta_{4} & =\frac{\mathbf{P}}{\mathbf{P}_{\text {ref }}}
\end{aligned}
$$

In principle, $\mathbf{P}_{\text {ref }}$ can be tailored to force the pressure to follow a desired thermodynamic path (e.g. a path that recovers the entropy flux from the parent three-dimensional flowfield). In this work, however, $\eta_{4}$ has been defined as unity (i.e. $\mathbf{P}_{\text {ref }}=\mathbf{P}$ ). This assumption is consistent with how the one-dimensional analysis codes that utilize this technique are typically exercised. Note that the momentum equation (a vector expression) has been reduced to a scalar equation by taking the dot product of this vector with the unit vector, $\overrightarrow{\mathbf{n}}$. Hence, the resulting uniform flow properties represent a true one-dimensionalization of the multi-dimensional parent flowfield, with the flow direction dictated by the choice of this unit vector. It can be shown that this methodology results in uniform properties that are analogous to those obtained based on the following operations:

- Area-weighting the pressure (assuming that $\eta_{4}=1$ )

- Mass flux-weighting the mean kinetic energy components

- Mass flux-weighting the static enthalpy

- Thermodynamic closure with an equation of state

\section{Conserved Mass/Energy/Entropy Method}

The CMES method (or thermodynamic state average) was first introduced by Riggins et al. ${ }^{8,9}$ The primary motivation behind this method was to address the deficiencies of the existing conserved-flux approaches 
as related to violations of the second law of thermodynamics. The CMME method (with or without distortion effects) introduces an entropy increase due solely to the "mixing loss" associated with the onedimensionalization process. The CMES method attempts to rectify this problem by explicitly conserving the entropy flux obtained from the parent multi-dimensional flowfield. Hence, this method results in uniform flow properties that satisfy the integral relations for conservation of mass, energy, and entropy, i.e.,

$$
\begin{aligned}
f_{\text {mass }}^{m} & =\left[\boldsymbol{\rho}(\overrightarrow{\mathbf{v}} \cdot \overrightarrow{\mathbf{n}}) \mathbf{Y}_{\mathbf{m}}\right] \mathbf{A} \\
f_{\text {energy }} & =[\boldsymbol{\rho}(\overrightarrow{\mathbf{v}} \cdot \overrightarrow{\mathbf{n}}) \mathbf{H}] \mathbf{A} \\
\int[\rho(\overrightarrow{\mathrm{v}} \cdot \vec{n}) s] d A=f_{\text {entropy }} & =[\boldsymbol{\rho}(\overrightarrow{\mathbf{v}} \cdot \overrightarrow{\mathbf{n}}) \mathbf{s}] \mathbf{A}
\end{aligned}
$$

The conservation of these fluxes ensures equivalency of mass addition, heating, and irreversible losses between the parent multi-dimensional flowfield and the one-dimensional flowfield. This statement holds regardless of the level (or type) of flow distortion that may be present because changes in mass, total enthalpy, and entropy are not influenced by flow distortion. Changes in these fluxes can only occur due to mass and/or heat addition (or extraction) and irreversible phenomena. The momentum flux, on the other hand, is affected by flow distortion, and its impact on the stream-thrust is accounted for in this method via the introduction of a single distortion parameter, $\eta$. This distortion parameter is defined in a manner that forces a match between the multi-dimensional stream-thrust and the one-dimensional value, i.e.,

$$
\int[\rho(\overrightarrow{\mathrm{v}} \cdot \vec{n}) \overrightarrow{\mathrm{v}}+P \vec{n}] \cdot \overrightarrow{\mathbf{n}} d A=\eta[\boldsymbol{\rho}(\overrightarrow{\mathbf{v}} \cdot \overrightarrow{\mathbf{n}}) \overrightarrow{\mathbf{v}} \cdot \overrightarrow{\mathbf{n}}+\mathbf{P}] \mathbf{A}
$$

Similar to the Langley distortion methodology, the CMES method produces a true one-dimensionalization of the multi-dimensional flow properties. The amount of distortion that is present in the flow is influenced by the choice of the unit vector, $\overrightarrow{\mathbf{n}}$. The procedure used to decode the one-dimensional flow properties for the CMES approach is given in Appendix B.

\section{Results}

Three examples have been compiled to illustrate various features associated with each of the onedimensionalization approaches considered in this effort. The first case involves the one-dimensionalization of a flowfield with an oblique shock wave. This example has an analytic solution with uniform flow properties in front of and behind the shock wave. Two grids were superimposed onto this flowfield. The first grid was aligned with the shock wave, while the second was representative of what would be used for CFD analyses. The second case considered was inviscid flow through a converging/diverging nozzle, and the third case was a representative scramjet isolator component. Cases 1 and 2 were specifically chosen to address issues associated with extracting one-dimensional parameters from multi-dimensional data sets. Case 3 was chosen to illustrate the performance of each averaging methodology for a representative high-speed propulsion component.

\section{Case 1}

The first case is an inviscid Mach 5 flow over a 20 degree compression corner. The analytic solution has uniform flow in front of the 29.8 degree shock wave and uniform flow behind the shock wave. The pre- and post-shock properties are given in Table 1 . The exact solution was one-dimensionalized along the cross-flow grid lines for each of the methods. Two different grids were used to determine the effect of grid topology on the one-dimensional properties. The first grid is shown in Fig. 2. This grid is composed of two blocks, divided by the shock wave, with grid lines that conform to the shock angle. The upper boundary of the solution domain represents a dividing streamline so that mass conservation is maintained. The two uniform flow regions, separated by the shock wave, can be seen in the Mach contours of Fig. 3.

Each one-dimensionalization strategy should be capable of returning the uniform flow properties provided by the multi-dimensional data set. Equation 1 shows that the weighted approach will always recover the correct uniform flow properties, since Eq. 1 reduces to

$$
\phi=\phi \frac{\int \mathrm{w} d A}{\int \mathrm{w} d A}=\phi
$$

4 of 19 
Table 1: Analytic Shock Properties

\begin{tabular}{|c|c|c|}
\hline Property & Pre-Shock & Post-Shock \\
\hline Pressure $[\mathrm{kPa}]$ & 101.325 & 713.066 \\
Temperature $[\mathrm{K}]$ & 300.0 & 636.8 \\
Density $\left[\mathrm{kg} / \mathrm{m}^{3}\right]$ & 1.1765 & 3.9007 \\
$\mathrm{u}[\mathrm{m} / \mathrm{s}]$ & 1736.2 & 1436.7 \\
$\mathrm{v}[\mathrm{m} / \mathrm{s}]$ & 0.0 & 522.9 \\
Mach number & 5.0 & 3.022 \\
\hline
\end{tabular}

when $\phi$ does not vary spatially. The properties returned by the conserved-flux approaches, however, are dependent upon how the unit normal, $\overrightarrow{\mathbf{n}}$, is defined. If the parent multi-dimensional data is uniform, then Eq. 2 reduces to

$$
\begin{aligned}
f_{\text {mass }}^{m} & =\left[\rho\left(\overrightarrow{\mathrm{v}} \cdot \int \vec{n} d A\right) Y_{m}\right] \\
\vec{f}_{\text {momentum }} & =\left[\rho\left(\overrightarrow{\mathrm{v}} \cdot \int \vec{n} d A\right) \overrightarrow{\mathrm{v}}+P \int \vec{n} d A\right] \\
f_{\text {energy }} & =\left[\rho\left(\overrightarrow{\mathrm{v}} \cdot \int \vec{n} d A\right) H\right]
\end{aligned}
$$

Comparing Eqs. 3 and 9 shows that the CMME method will return the correct uniform flow properties provided that $\overrightarrow{\mathbf{n}}$ is defined as

$$
\overrightarrow{\mathbf{n}}=\frac{\int \vec{n} d A}{\mathbf{A}}
$$

Another choice for the unit vector that will recover the correct uniform flow properties is one aligned with the velocity vector. In this scenario, a scalar momentum equation (stream-thrust) is formed by taking the dot product of the momentum vector with $\overrightarrow{\mathbf{n}} \equiv \overrightarrow{\mathbf{v}} /|\overrightarrow{\mathbf{v}}|$. This step aligns the momentum with the direction of the flow velocity, and results in an equation set that is locally one-dimensional. Any other choice for the unit normal will not reproduce the analytical data for this flow. This feature is illustrated in Fig. 4 which compares the Mach number obtained by the CMME method based on the unit normal given by Eq. 10, with that of one chosen to be aligned with the $x$-coordinate. The Mach number obtained by the weighted approach is also shown for reference purposes. The use of a unit normal aligned with the $x$-coordinate removes the contributions from the $y$-momentum equation during the decomposition process. This "loss" of momentum prevents the matching of properties downstream of the shock. It should be noted that the distance variable used to plot the one-dimensional data is defined as the average of the $x$-coordinate along the line (or plane) of integration.

The choice made for the unit normal has no effect on the flow variables extracted from the Langley distortion methodology, but it will affect the values obtained for the distortion coefficients. The distortion coefficients will be unity (for this uniform flow case) only if the unit normal is defined to be parallel to the velocity direction. Any other choice will result in non-unity distortion coefficients due to the lack of alignment with the velocity field. The CMES method does not contain a vector flux quantity, and velocity only appears in the form of $(\overrightarrow{\mathbf{v}} \cdot \overrightarrow{\mathbf{n}})$. The decomposition process for this approach requires the evaluation of the kinetic energy (i.e. velocity magnitude). Thus, the only logical choice for the unit normal is one that is aligned with the velocity vector, since this choice results in $(\overrightarrow{\mathbf{v}} \cdot \overrightarrow{\mathbf{n}})=\sqrt{\overrightarrow{\mathbf{v}} \cdot \overrightarrow{\mathbf{v}}}$. The distortion coefficient is also unity (as it should be for uniform flow) with the unit normal chosen in this manner. Since $(\overrightarrow{\mathbf{v}} \cdot \overrightarrow{\mathbf{n}})$ is the only velocity information available with this approach, the velocity components for flow alignment must be supplied by some external means (such as mass flux-weighting). Figure 5 verifies that the Langley distortion methodology and the CMES method (with the unit normal aligned with the velocity direction) recover the parent uniform properties. Although not shown, the distortion coefficients are all unity. Based on the observations outlined above, all remaining properties based on the CMME method will utilize a unit normal based on Eq. 10, and a unit normal aligned with the velocity vector will be used for the Langley distortion and CMES methods. 
The second grid, shown in Fig. 6, is typical of a grid topology that would be used in a shock-capturing CFD simulation. The grid has a single block with vertical grid lines in the cross-flow direction. Hence, the distance variable in all of the uniform flow property plots will precisely coincide with each streamwise integration plane. The area-weighted and mass flux-weighted properties $(\rho, u, v$, and $P)$ for this grid are given in Figs. 7 and 8. Both averaging methods give the exact pre- and post-shock values for grid lines in regions of uniform flow (i.e. near the inflow and outflow planes). Along grid lines passing through both preshock and post-shock flow, the two methods give significantly different values. For this problem, a weighted property can be written in the following form,

$$
\phi=\left(\phi_{1}-\phi_{2}\right) G+\phi_{2}
$$

where

$$
G=\frac{\mathrm{w}_{1} A_{1}}{\left(\mathrm{w}_{1} A_{1}+\mathrm{w}_{2} A_{2}\right)}
$$

and the subscripts 1 and 2 refer to the pre- and post-shock properties, respectively. For area-weighting (i.e. $\mathrm{w}=1), G$ reduces to

$$
G=\frac{y_{\max }-x \tan \beta}{y_{\max }-x \tan \theta} \quad \text { for } 0 \leq x \leq \frac{y_{\max }}{\tan \beta}
$$

where $y_{\max }$ is the height of the inflow streamline, $\beta$ is the shock angle, and $\theta$ is the turning angle. This is a non-linear relationship in $x$ and gives the curved distributions shown in the figures. For mass flux-weighting, i.e. $\mathrm{w}=\rho(\vec{v} \cdot \vec{n}), G$ reduces to

$$
G=\frac{\rho_{1} u_{1}\left(y_{\max }-x \tan \beta\right)}{\dot{m}} \quad \text { for } 0 \leq x \leq \frac{y_{\max }}{\tan \beta}
$$

The mass flow rate is conserved, so $\dot{m}$ is constant leading to a relationship that is linear in $x$. As a result, all of the mass flux-weighted properties vary linearly for integration planes that cross the shock wave.

There are two approaches that can be used to compute a set of weighted properties. The first is to simply weight every property of interest independently. The second is to weight only a minimum number of properties, and compute all additional properties using thermodynamic and gas-dynamic relationships. These two approaches are illustrated in Fig. 9, which shows the Mach number and total pressure distributions using both approaches. The solid and dashed lines are the mass flux-weighted and area-weighted distributions, while the symbols are the distributions computed from the weighted density, velocity, and pressure. The weighted Mach numbers and total pressures follow the behavior defined above, whereas the values computed from the minimum set of weighted averages are significantly different. This is a direct result of the non-linear relationships between the flow properties. For this particular minimum set of properties, the total pressure distribution shows a local minimum, which is a violation of the second law of thermodynamics. Somewhat different results would be obtained if a another set of weighted properties had been chosen.

The property distributions computed with the CMME, Langley distortion, CMES, and mass flux-weighted methods are plotted in Figs. 10 and 11. The CMME, Langley distortion, and CMES methods give similar results for the static pressure distribution, but each of these deviate significantly from the mass flux-weighted values. The $x$-velocity distributions are similar for all approaches except for the values obtained from the CMES method. Interestingly, the Mach number obtained from the CMES method compares favorably with the mass flux-weighted Mach number; indicating that the static temperature given by this method is greater than the mass flux-weighted value. The total pressure distributions that result from the CMME and Langley distortion methods have a local minimum, similar to what was observed with the weighted averages when the weighting process was not performed independently. The CMES method does not show this behavior for the total pressure because the entropy, which is closely coupled to the total pressure, is one of the quantities that was conserved.

\section{Case 2}

The second case is a two-dimensional inviscid flow through a converging/diverging nozzle with an exit Mach number of 3. The contour was generated with the IMOCND (Irrotational Method of Characteristics for Nozzle Design) code, ${ }^{12}$ and has a throat defined by a Gaussian curve with a radius of curvature equal to one half of the throat height. The grid and Mach contours for this flowfield are shown in Fig. 12. It is common 
practice in CFD to take advantage of any symmetry present in the geometry to reduce the time required to compute steady-state solutions. This convention can have an affect on the properties computed by the various one-dimensionalization methods. Figure 13 shows the distributions of the $y$-component of velocity computed from the full nozzle solution and the solution of just the upper half of the nozzle. When only the upper half of the geometry is processed, all of the methods produce a negative $y$-velocity distribution upstream of the throat (where the flow is turned towards the centerline), and a positive $y$-velocity distribution downstream of the throat (where the flow is turned away from the centerline). When the complete geometry is processed, all of the methods produce a zero cross-flow velocity except for the Langley distortion method. The Langley distortion method extracts the velocity magnitudes from the kinetic energy fluxes, i.e.,

$$
\begin{aligned}
\mathbf{u}^{2} & =\frac{\int \rho(\overrightarrow{\mathrm{v}} \cdot \vec{n}) u^{2} d A}{\dot{m}} \\
\mathbf{v}^{2} & =\frac{\int \rho(\overrightarrow{\mathrm{v}} \cdot \vec{n}) v^{2} d A}{\dot{m}} \\
\mathbf{w}^{2} & =\frac{\int \rho(\overrightarrow{\mathrm{v}} \cdot \vec{n}) w^{2} d A}{\dot{m}}
\end{aligned}
$$

Hence, there exists no cancellation of velocity components across the line of symmetry, as is the case for each of the other methods. Note that this method also produces larger $y$-velocity components than the other methods (for the half geometry). This behavior can be explained by comparing the expressions for the weighted average of velocity

$$
\mathbf{v}=\left(\frac{\sum_{n=1}^{n p t s} w_{n} v_{n}}{\sum_{n=1}^{n p t s} w_{n}}\right) \approx \frac{1}{n p t s} \sum_{n=1}^{n p t s} v_{n}
$$

with that of the square root of the weighted average of the velocity squared

$$
\mathbf{v}=\left(\frac{\sum_{n=1}^{n p t s} w_{n} v_{n}^{2}}{\sum_{n=1}^{n p t s} w_{n}}\right)^{\frac{1}{2}} \approx\left(\frac{1}{n p t s} \sum_{n=1}^{n p t s} v_{n}^{2}\right)^{\frac{1}{2}}
$$

In the above expressions, it has been assumed that the weighting factors are approximately equal at each point. A close examination of these expressions reveals that Eq. 17 produces velocity values that are greater than or equal to those obtained from Eq. 16.

Other than the $y$-velocity component, the weighted averages obtained from the full nozzle match those of the half nozzle. The flux-conserved methods, on the other hand, involve a system of coupled equations. Hence, a change in any one property can affect other properties. Figures 13 and 14 show the distributions of various properties through the nozzle for each of the one-dimensionalization approaches. The largest differences between full and half geometry properties occur when the CMME method is utilized. Both the Mach number and total pressure show noticeable deviations from the values obtained with the other averaging approaches. The total pressure distributions are particularly interesting because this quantity should be constant for the isentropic flowfield considered here. The CMME value for the full nozzle solution shows nearly a $20 \%$ total pressure drop in regions where the $y$-component of velocity is large. The half nozzle solution also shows a drop in total pressure, but the drop is considerably smaller (approximately $5 \%$ ). The larger total pressure drop seen with the full nozzle is a result of an inconsistent accounting of the kinetic energy between the three-dimensional and one-dimensional spaces. The kinetic energy computed from the full nozzle solution only includes contributions from the $x$-velocity component (the $y$-component vanishes), leading to a value for the kinetic energy that is smaller than that computed for the half nozzle. Total enthalpy is one of the properties that is conserved, so the static enthalpy (or static temperature):

$$
\mathbf{h}\left(\mathbf{T}, \mathbf{Y}_{\mathbf{m}}\right)=\mathbf{H}-\frac{1}{2}(\overrightarrow{\mathbf{v}} \cdot \overrightarrow{\mathbf{v}})
$$

will be larger for the full nozzle. This larger temperature results in the smaller Mach number and total pressure values seen in Fig. 14. The results obtained with the CMME method, and to a lesser extent the Langley distortion methodology also show a violation of the second law of thermodynamics. This undesirable behavior is avoided when the weighted average or the CMES method is employed. 


\section{Case 3}

The third case is a two-dimensional, turbulent flow through a model scramjet isolator. The inflow conditions simulated are given in Table 2, along with the assumed surface temperature and applied back-pressure. The lower wall of the isolator is part of the facility flowpath, while the upper wall is a flat plate extension placed into the test section. This produces an asymmetric boundary layer structure, providing a more realistic simulation of what would occur with a flight engine. The back-pressure was set high enough to force a shock system into the isolator, simulating the pre-combustion shock train flow physics of a scramjet operating in dual-mode. The grid and Mach contours of the back-pressured isolator flowfield are shown in Fig. 15 . As one would expect, the thicker boundary layer on the lower surface separated to a larger extent than the adjacent boundary layer on the upper surface. The CFD simulation for this configuration was performed using the VULCAN flow solver ${ }^{13,14}$ with the Wilcox (1998) $k$-w turbulence model. ${ }^{15}$ The turbulent kinetic energy is a contributor to the total enthalpy flux, and the decomposition required for the one-dimensionalization methods took this into account, i.e.

$$
\mathbf{H}=\mathbf{h}\left(\mathbf{T}, \mathbf{Y}_{\mathbf{m}}\right)+\frac{1}{2}(\overrightarrow{\mathbf{v}} \cdot \overrightarrow{\mathbf{v}})+\mathbf{k}
$$

where

$$
\int[\rho(\overrightarrow{\mathrm{v}} \cdot \vec{n}) k] d A=f_{t k e}=[\boldsymbol{\rho}(\overrightarrow{\mathbf{v}} \cdot \overrightarrow{\mathbf{n}}) \mathbf{k}] \mathbf{A}
$$

Table 2: Isolator Conditions

\begin{tabular}{||c|c|}
\hline \hline Nominal Isolator Conditions & Inflow \\
\hline \hline Mach Number & 2.22 \\
\hline Total Temperature $[\mathrm{K}]$ & 286.1 \\
\hline Total Pressure [kPa] & 923.9 \\
\hline Surface Temperature [K] & 250.0 \\
\hline Back Pressure [kPa] & 400.0 \\
\hline \hline
\end{tabular}

The shock system in the isolator introduces a high level of flow distortion; presenting a significant challenge for any one-dimensionalization methodology. Figure 16 shows the distributions of Mach number and total pressure through the isolator for the CMME, Langley distortion, CMES, and mass flux-weighted approaches. The oblique shock pattern in the isolator causes a reduction in Mach number from a supersonic condition at the isolator entrance, to a subsonic condition near the exit of the isolator. The cross-sectional area in the isolator, however, is constant. Under these circumstances, the CMME method relationships (Eq. 3) can permit only two solutions: a supersonic (shock-free) solution and a subsonic (normal-shock) solution. Hence, this one-dimensionalization approach is not capable of predicting the gradual compression through the isolator. The Langley distortion method includes distortion coefficients that allow for an "effective" area change; providing a mechanism for capturing the effects of the oblique wave patterns through the isolator. The shock wave patterns in the Mach distribution obtained from this approach match those of the mass flux-weighted Mach distribution quite well. The Mach number extracted with the CMES method exhibits a behavior similar to that given by the CMME approach. However, since entropy is a quantity that is explicitly matched, a physically consistent monotonic drop in total pressure is realized with this methodology. Both of the CMME methodologies predict a non-monotonic change in total pressure. This behavior has been a consistent trend for each test case when non-negligible levels of flow distortion are present. This observation (to a large degree) motivated the development of the CMES methodology.

The distortion coefficients extracted from the Langley distortion and CMES methods are given in Fig. 17. The $\eta_{3}$ distortion parameter (present in the Langley distortion method) never deviates from unity. This is a direct result of choosing the unit normal to be aligned with the velocity vector. All other distortion parameters deviate significantly from unity. The gradual reduction seen for the $\eta_{1}$ and $\eta_{2}$ distortion parameters upstream of the shock system is primarily a boundary layer displacement effect. The onset of the shock system causes a rapid drop in these distortion parameters, and they remain low until the flow has been shocked down to subsonic conditions. The $\eta_{1}$ and $\eta_{2}$ parameters gradually rise downstream of the shock system as 
the flow attempts to become re-attached. The distortion parameter present in the CMES method, $\eta$, shows a gradual rise through shock system up to the point where the subsonic solution (see Fig. 16) is achieved. At this point, the distortion parameter drops discontinuously and gradually increases for the remainder of the re-attachment process.

\section{Conclusions}

Issues involved with the extraction of uniform properties from experimental data sets and/or multidimensional computational data sets have been examined for a variety of methodologies. The unique assumptions associated with each one-dimensionalization approach were shown to significantly affect the values computed by each method. In general, the conserved-flux approaches all had difficulty producing expected one-dimensional flow properties when the primary flow direction was not uniform. The conserved-flux approaches that did not explicitly account for the entropy flux were particularly troublesome, since these methods were susceptible to violations of the second law of thermodynamics. The assumption of symmetry often used in CFD analysis was also shown to have an impact on the one-dimensionalization process. In general, the assumption of symmetry helped to maintain the correct kinetic energy levels for the conservedflux methodologies, which led to an improved uniform flow representation of the nozzle flowfields studied in this effort. Finally, while no general arguments can be given for one averaging approach over another, some basic observations can be made for each class of approach. The weighted methods, particularly the mass flux-weighted approach, consistently predicted the qualitative trends present in the multi-dimensional data. As a result, this approach is attractive when one simply wants to qualitatively examine how properties are varying through the flowpath. The shortcoming of this approach appears when one wants to interface with a one-dimensional engineering tool (or in any other situation where strict consistency between the flow properties is required). In this situation, the approach used to one-dimensionalize the data should be consistent with the fluxes used by the engineering tool, and the interface should be placed at a plane with minimal flow distortion.

\section{References}

${ }^{1}$ Billig, F. S., "Design of Supersonic Combustors Based on Pressure-Area Fields," Proceedings of the Eleventh Symposium (International) on Combustion, Pittsburgh, Pa., April 1967, pp. 755-769.

${ }^{2}$ Pandolfini, P. P., Billig, F. S. Corpening, G. P., Corda, S. and Friedman, M. A., "Analyzing Hypersonic Engines Using the Ramjet Performance Analysis Code," APL Technical Review, Vol. Vol. 2, No. 1, 1990, pp. 68-78.

${ }^{3}$ Pinckney, S. Z., "Internal Performance Predictions for Langley Scramjet Engine Module," NASA TM X-74038, 1978.

${ }^{4}$ Pandolfini, P. P. and Friedman, M. A., "Instructions for Using Ramjet Performance Analysis Code RJPA," Tech. Rep. AL-92-P175, JHU/APL, June 1992.

${ }^{5}$ Tsai, C.-Y. and Rizkalla, S., "GASL1D: A 1-D Combustor Design and Analysis Code," GASL TM 261, General Applied Science Laboratories, Inc., Feb. 1995.

${ }^{6}$ Pinckney, S. Z., Ferlemann, S. M., Mills, G., and Takashima, N., "Program Manual for SRGULL Version 1.0: Second Generation Engineering Model for the Prediction of Airframe-Integrated Subsonic/Supersonic Combustion Ramjet Cycle Performance," Hyper-X 829, July 2000.

${ }^{7}$ Auslender. A. H., "An Application of Distortion Analysis to Scramjet-Combustor Performance Assessment," Scramjet Engine Performance Analysis, Evaulation, and Optimization - JANNAF Propulsion and Joint Subcommittee Scramjet Performance Workshop, Dec. 1996.

${ }^{8}$ Riggins, D. W., McClinton, C. R., and Vitt, P. H., "Thrust Losses in Hypersonic Engines Part 1: Methodology," Journal of Propulsion and Power, Vol. Vol. 13, No. 2, March-April 1997, pp. 281-287.

${ }^{9}$ Riggins, D. W., "Thrust Losses in Hypersonic Engines Part 2: Applications," Journal of Propulsion and Power, Vol. Vol. 13, No. 2, March-April 1997, pp. 288-295.

${ }^{10}$ Wyatt, D. D., "Analysis of Errors Introduced by Several Methods of Weighting Nonuniform Duct Flows," NASA Technical Note $3400,1955$.

${ }^{11}$ McLafferty, G. H., "A Generalized Approach to the Definition of Average Flow Quantities in Nonuniform Streams," United Aircraft Corp. R-13534, 1954.

${ }^{12}$ Gaffney, R. L., Stewart, B. K., and Harvin, S. F., "The Design of a High-Q Mach-5 Nozzle for the NASA 8-Foot HTT," AIAA Paper 2006-2954, June 2006.

${ }^{13}$ White, J. A. and Morisson, J. H., "Pseudo-Temporal Multi-Grid Relaxation Scheme for Solving the Parabolized NavierStokes Equations," AIAA Paper 99-3360, June 1999.

${ }^{14}$ VULCAN, "http://vulcan-cfd.larc.nasa.gov/," Jan. 2007.

${ }^{15}$ Wilcox, D. C., Turbulence Modeling for CFD, DCW Industries, Inc., 2nd ed., 1998. 


\section{Appendix A. CMME Method - Variable Decomposition}

The uniform flow properties that satisfy the integral flux relations for mass, momentum, and energy conservation can be written as

$$
\begin{aligned}
f_{\text {mass }}^{m} & =\left[\boldsymbol{\rho}(\overrightarrow{\mathbf{v}} \cdot \overrightarrow{\mathbf{n}}) \mathbf{Y}_{\mathbf{m}}\right] \mathbf{A} \\
\vec{f}_{\text {momentum }} & =[\boldsymbol{\rho}(\overrightarrow{\mathbf{v}} \cdot \overrightarrow{\mathbf{n}}) \overrightarrow{\mathbf{v}}+\mathbf{P} \overrightarrow{\mathbf{n}}] \mathbf{A} \\
f_{\text {energy }} & =[\boldsymbol{\rho}(\overrightarrow{\mathbf{v}} \cdot \overrightarrow{\mathbf{n}}) \mathbf{H}] \mathbf{A}
\end{aligned}
$$

The introduction of the total mass flux, $\dot{m}$,

$$
\dot{m}=\sum_{m=1}^{n s} f_{m a s s}^{m}
$$

allows these relations to be recast as

$$
\begin{aligned}
f_{\text {mass }}^{m} & =\dot{m} \mathbf{Y}_{\mathbf{m}} \\
\vec{f}_{\text {momentum }} & =\dot{m} \overrightarrow{\mathbf{v}}+\mathbf{P} \overrightarrow{\mathbf{n}} \mathbf{A} \\
f_{\text {energy }} & =\dot{m} \mathbf{H}
\end{aligned}
$$

The mass expression provides an explicit relationship for the uniform composition variables,

$$
\mathbf{Y}_{\mathbf{m}}=\frac{f_{m a s s}^{m}}{\dot{m}}
$$

and the energy expression provides a direct relationship for the uniform total enthalpy

$$
\mathbf{H}=\frac{f_{\text {energy }}}{\dot{m}}=\mathbf{h}\left(\mathbf{T}, \mathbf{Y}_{\mathbf{m}}\right)+\frac{1}{2}(\overrightarrow{\mathbf{v}} \cdot \overrightarrow{\mathbf{v}})
$$

The momentum expression can be rearranged to yield an expression for the velocity vector,

$$
\overrightarrow{\mathbf{v}}=\frac{\vec{f}_{\text {momentum }}-\mathbf{P} \overrightarrow{\mathbf{n}} \mathbf{A}}{\dot{m}}
$$

This expression can be simplified to a scalar equation by taking the dot product of this vector with the unit normal $(\overrightarrow{\mathbf{n}})$,

$$
\overrightarrow{\mathbf{v}} \cdot \overrightarrow{\mathbf{n}}=\frac{f_{\text {momentum }}-\mathbf{P A}}{\dot{m}}
$$

where

$$
f_{\text {momentum }} \equiv\left(\vec{f}_{\text {momentum }} \cdot \overrightarrow{\mathbf{n}}\right)
$$

Further manipulations are possible with the introduction of the equation of state,

$$
\mathbf{P}=\rho \mathbf{R T}=\frac{\dot{m} \mathbf{R T}}{(\overrightarrow{\mathbf{v}} \cdot \overrightarrow{\mathbf{n}}) \mathbf{A}}
$$

resulting in the following relationship for $(\overrightarrow{\mathbf{v}} \cdot \overrightarrow{\mathbf{n}})$

$$
\overrightarrow{\mathbf{v}} \cdot \overrightarrow{\mathbf{n}}=\frac{f_{\text {momentum }}-\mathbf{P A}}{\dot{m}}=\frac{f_{\text {momentum }}}{\dot{m}}-\frac{\mathbf{R T}}{(\overrightarrow{\mathbf{v}} \cdot \overrightarrow{\mathbf{n}})}
$$

This equation is quadratic with respect to $(\overrightarrow{\mathbf{v}} \cdot \overrightarrow{\mathbf{n}})$, hence the quadratic formula can be used to obtain:

$$
\overrightarrow{\mathbf{v}} \cdot \overrightarrow{\mathbf{n}}=\frac{f_{\text {momentum }} / \dot{m} \pm\left[\left(f_{\text {momentum }} / \dot{m}\right)^{2}-4 \mathbf{R T}\right]^{\frac{1}{2}}}{2}
$$

Equations 4, 5, 6, 9 and 11 can be combined to yield a single expression with static temperature as the only unknown. 
In principle, any root solving algorithm can be used to solve for the static temperature. Unfortunately, this function is multi-valued with respect to temperature, requiring additional logic and/or information in order to choose the desired root for a given set of fluxes. The function, $F(T)$, has the general shape displayed in Fig. A1. This figure shows that two values of temperature can satisfy $F(T)=0$. In this effort, two bisection solves are performed to determine each temperature value that satisfies $F(T)=0$. The first bisection method finds the root that is bounded between $\left[T_{\min }, T_{F_{\max }}\right]$, and the second finds the root that is bounded between $\left[T_{F_{\max }}, T_{\max }\right] . T_{F_{\max }}$ is the temperature that maximizes the function $F(T)$. This temperature can be found from the solution of $d F / d T=0$. The maximum allowable temperature $\left(T_{\max }\right)$ is the temperature that forces the discriminant of Eq. 11 to be zero, i.e.,

$$
\mathbf{T}=\frac{\left(f_{\text {momentum }} / \dot{m}\right)^{2}}{4 \mathbf{R}}
$$

and $T_{\min }$ can be taken as either zero, or the minimum temperature specified for the polynomial fits of the thermodynamic data. The solution that is retained is the solution that yields a Mach number that lies closest to the mass flux-weighted Mach number, which must be externally supplied. As a final note, one may be tempted to assume that the two roots that satisfy $F(T)=0$ represent a subsonic and a supersonic solution to the flux equations. A careful examination of Eq. 11 reveals that this is not necessarily the case. The Mach number (based on $\overrightarrow{\mathbf{v}} \cdot \overrightarrow{\mathbf{n}}$ ) that appears when the discriminant vanishes corresponds to a value of $1 / \sqrt{\gamma}$, where $\gamma$ is the ratio of specific heats. Hence, the two temperature values that satisfy $F(T)=0$ can correspond to two subsonic solutions, or a subsonic and a supersonic solution.

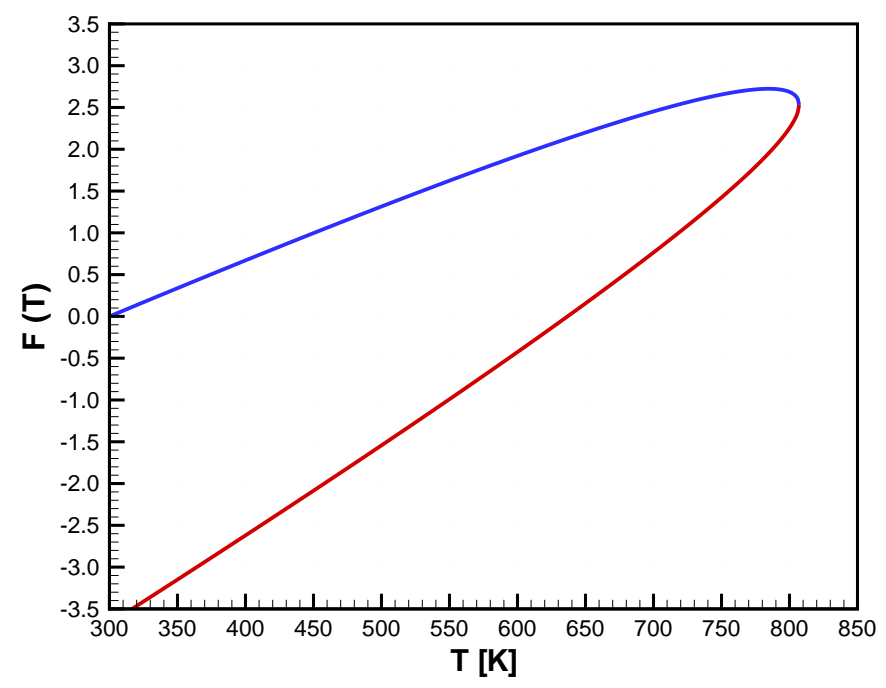

Figure A1: General form of the CMME function (colored lines denote the two branches of Eq. 11) 


\section{Appendix B. CMES Method - Variable Decomposition}

The uniform flow properties that satisfy the integral flux relations for mass, energy, and entropy conservation can be written as

$$
\begin{aligned}
f_{\text {mass }}^{m} & =\left[\boldsymbol{\rho}(\overrightarrow{\mathbf{v}} \cdot \overrightarrow{\mathbf{n}}) \mathbf{Y}_{\mathbf{m}}\right] \mathbf{A} \\
f_{\text {energy }} & =[\boldsymbol{\rho}(\overrightarrow{\mathbf{v}} \cdot \overrightarrow{\mathbf{n}}) \mathbf{H}] \mathbf{A} \\
f_{\text {entropy }} & =[\boldsymbol{\rho}(\overrightarrow{\mathbf{v}} \cdot \overrightarrow{\mathbf{n}}) \mathbf{s}] \mathbf{A}
\end{aligned}
$$

The introduction of the total mass flux, $\dot{m}$,

$$
\dot{m}=\sum_{m=1}^{n s} f_{m a s s}^{m}
$$

allows these relations to be recast as

$$
\begin{aligned}
f_{\text {mass }}^{m} & =\dot{m} \mathbf{Y}_{\mathbf{m}} \\
f_{\text {energy }} & =\dot{m} \mathbf{H} \\
f_{\text {entropy }} & =\dot{m} \mathbf{s}\left(\mathbf{T}, \mathbf{P}, \mathbf{Y}_{\mathbf{m}}\right)
\end{aligned}
$$

leading to explicit relationships for the uniform composition variables, total enthalpy, and entropy. If the unit normal is defined to be aligned with the velocity vector $(i . e ., \overrightarrow{\mathbf{n}}=\overrightarrow{\mathbf{v}} /|\overrightarrow{\mathbf{v}}|)$, then

$$
(\overrightarrow{\mathbf{v}} \cdot \overrightarrow{\mathbf{n}})=\sqrt{\overrightarrow{\mathbf{v}} \cdot \overrightarrow{\mathbf{v}}}
$$

allowing $(\overrightarrow{\mathbf{v}} \cdot \overrightarrow{\mathbf{n}})$ to be extracted from the definition of total enthalpy,

$$
(\overrightarrow{\mathbf{v}} \cdot \overrightarrow{\mathbf{n}})=\left[2\left(\mathbf{H}-\mathbf{h}\left(\mathbf{T}, \mathbf{Y}_{\mathbf{m}}\right)\right)\right]^{\frac{1}{2}}
$$

It should be noted that the velocity vector is not present in the equations that govern the CMES method. Therefore, it must be supplied by some other means (e.g. by mass flux-weighting the velocity components) to define the unit normal. Finally, an expression for the pressure is obtained by combining the equation of state with the total mass flux, i.e.

$$
\mathbf{P}=\rho \mathbf{R T}=\frac{\dot{m} \mathbf{R T}}{(\overrightarrow{\mathbf{v}} \cdot \overrightarrow{\mathbf{n}}) \mathbf{A}}
$$

Equations 3, 5, and 6 can be combined to yield a single expression with static temperature as the only unknown. The resulting function, $F(T)$, has the general shape displayed in Fig. B1. This figure shows that two values of temperature can satisfy $F(T)=0$. One of the roots results in a solution for subsonic flow, and the other yields a solution for supersonic flow. In this effort, two bisection solves are performed to determine each temperature value that satisfies $F(T)=0$. The first bisection method finds the root that is bounded between $T_{\min }$ and the temperature at the sonic point, and the second finds the root that is bounded between the temperature at the sonic point and the stagnation temperature. The stagnation temperature is obtained from the solution of

$$
\mathbf{H}\left(\mathbf{T}_{\circ}, \mathbf{Y}_{\mathbf{m}}\right)=0
$$

the sonic temperature is the temperature that satisfies

$$
\gamma\left(\mathbf{T}^{*}, \mathbf{Y}_{\mathbf{m}}\right) \mathbf{R} \mathbf{T}^{*}=2\left(\mathbf{H}-\mathbf{h}\left(\mathbf{T}^{*}, \mathbf{Y}_{\mathbf{m}}\right)\right)
$$

and $T_{\min }$ can be taken as either zero, or the minimum temperature given for the polynomial fits of the thermodynamic data. The solution that is retained is the solution that yields a Mach number that lies closest to the mass flux-weighted Mach number (which must be externally supplied). 


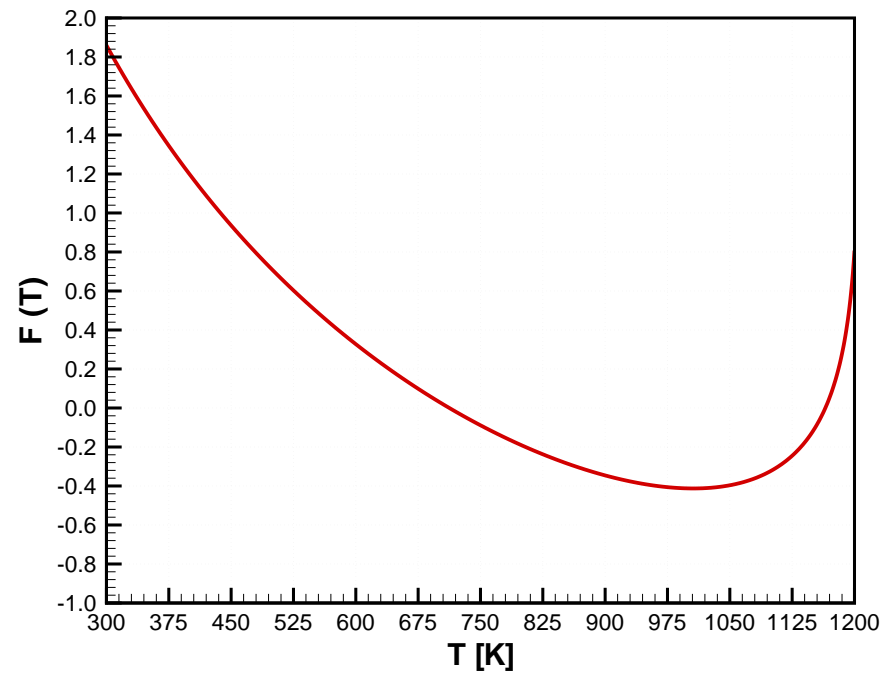

Figure B1: General form of the CMES function

\section{Figures}

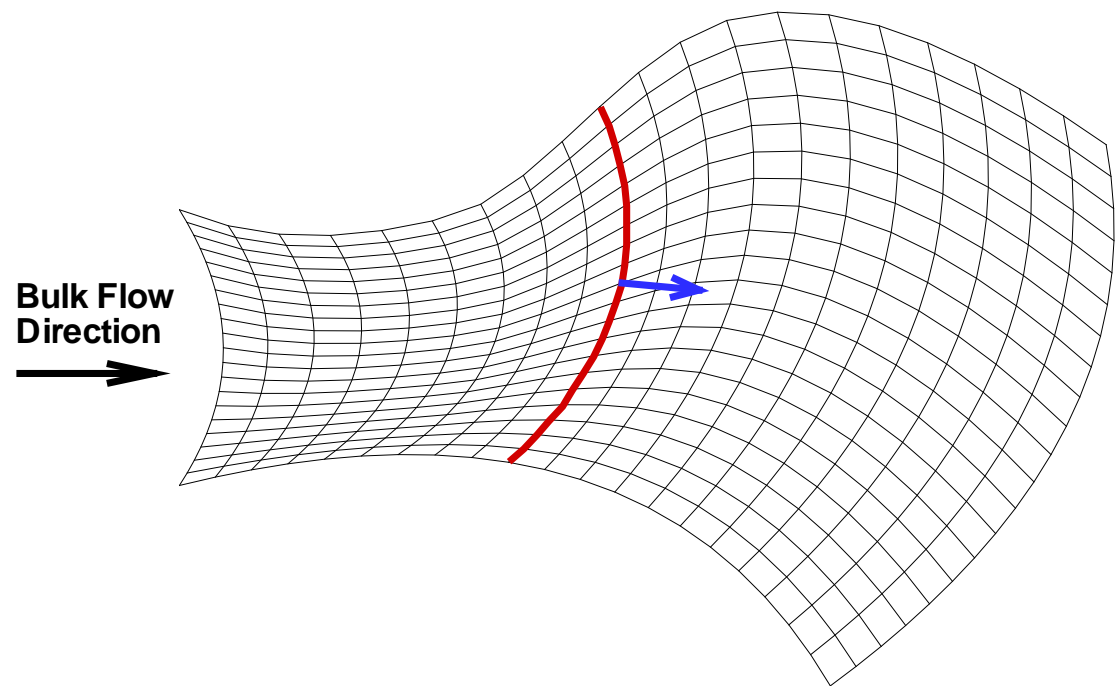

Figure 1: Surface/Line of integration and the unit normal for one-dimensionalization 


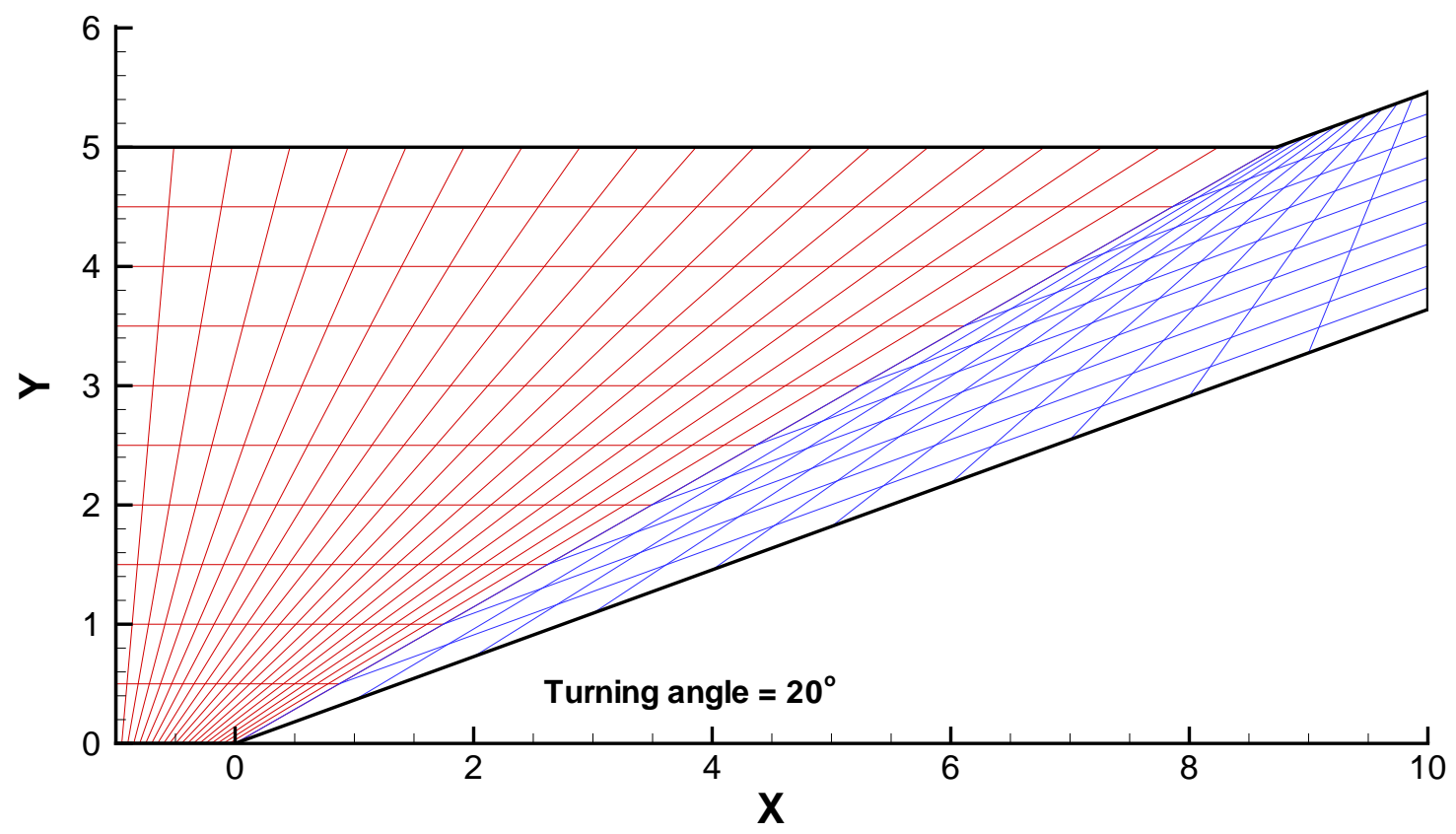

Figure 2: Grid 1 - Two blocks aligned with the shock wave

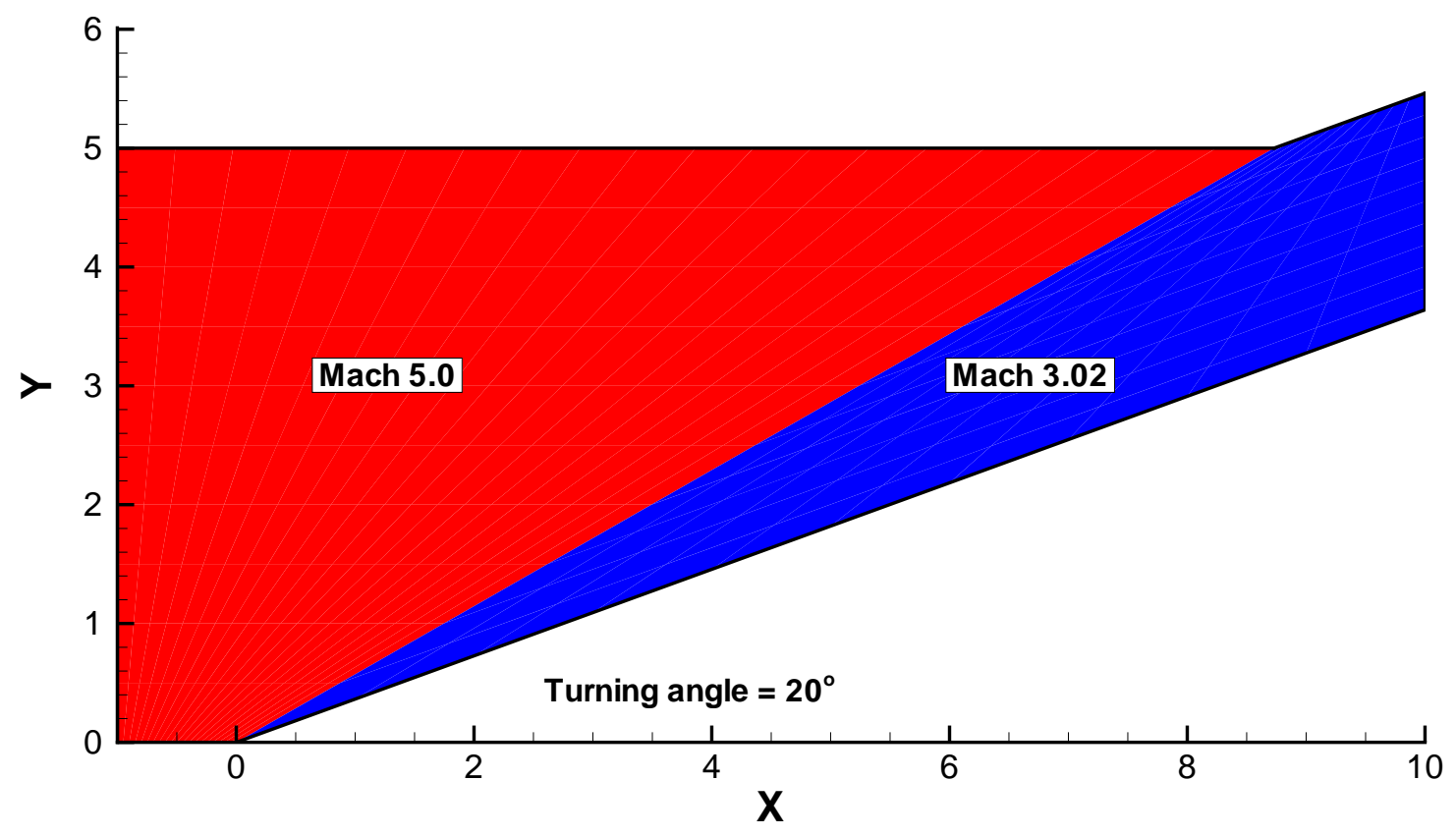

Figure 3: Mach number contours for the compression corner 

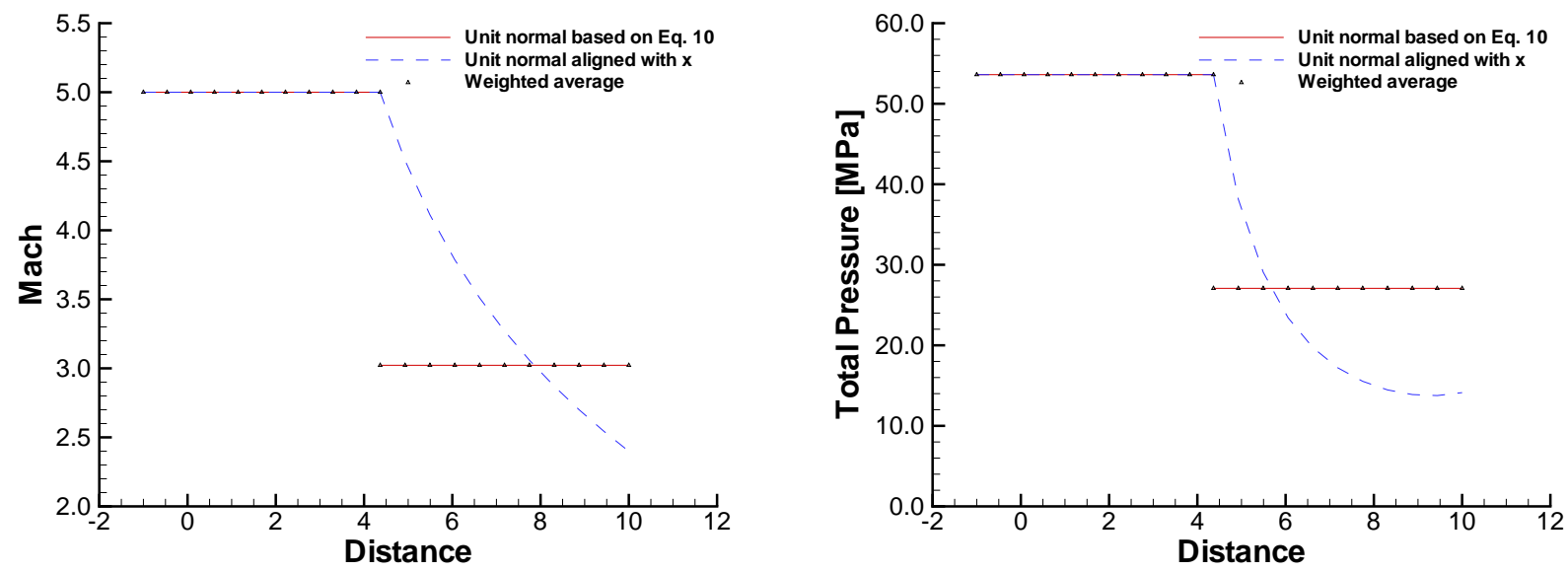

Figure 4: Variation of uniform CMME properties with choice of unit normal (the distance variable is defined as the average $x$-value along a given integration surface)
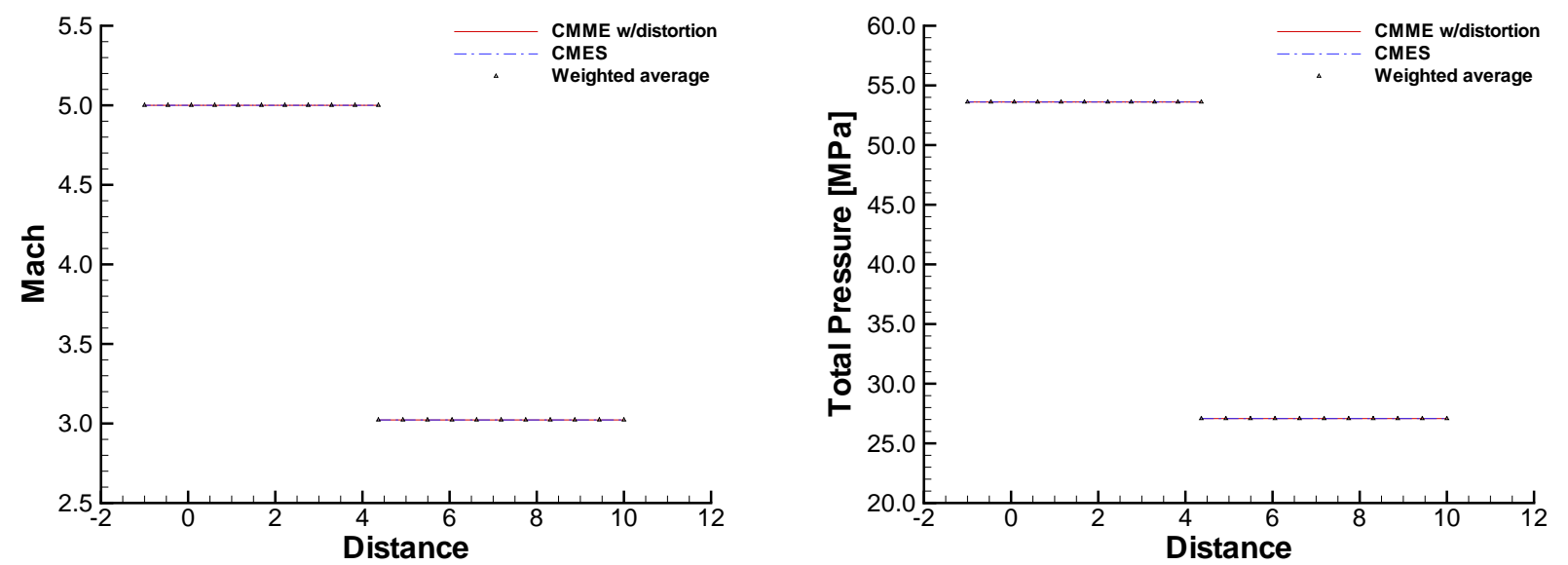

Figure 5: Uniform properties obtained with the Langley distortion and CMES methodologies (the distance variable is defined as the average $x$-value along a given integration surface)

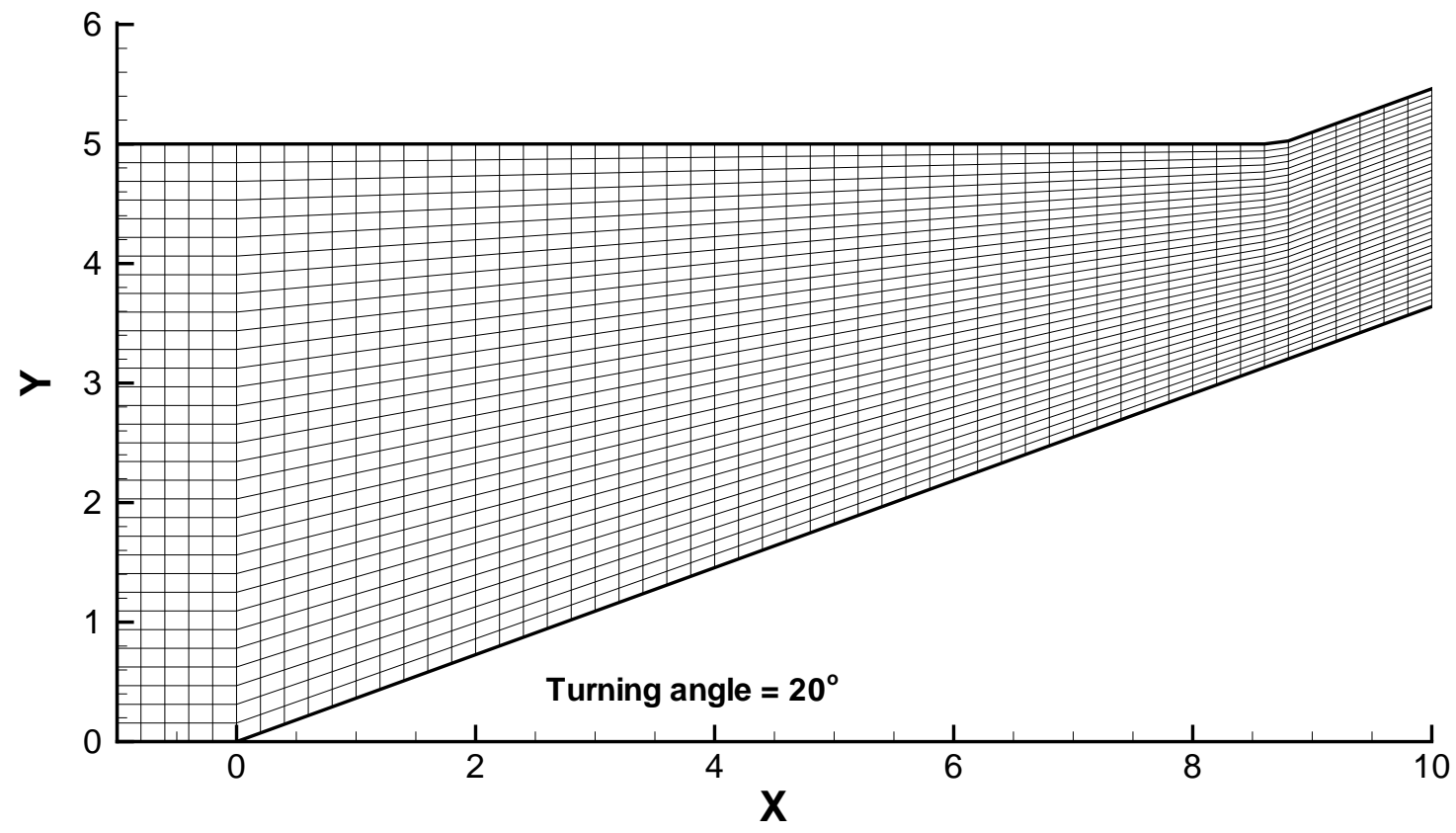

Figure 6: Grid 2 - Single block typical of a grid used for CFD

15 of 19 

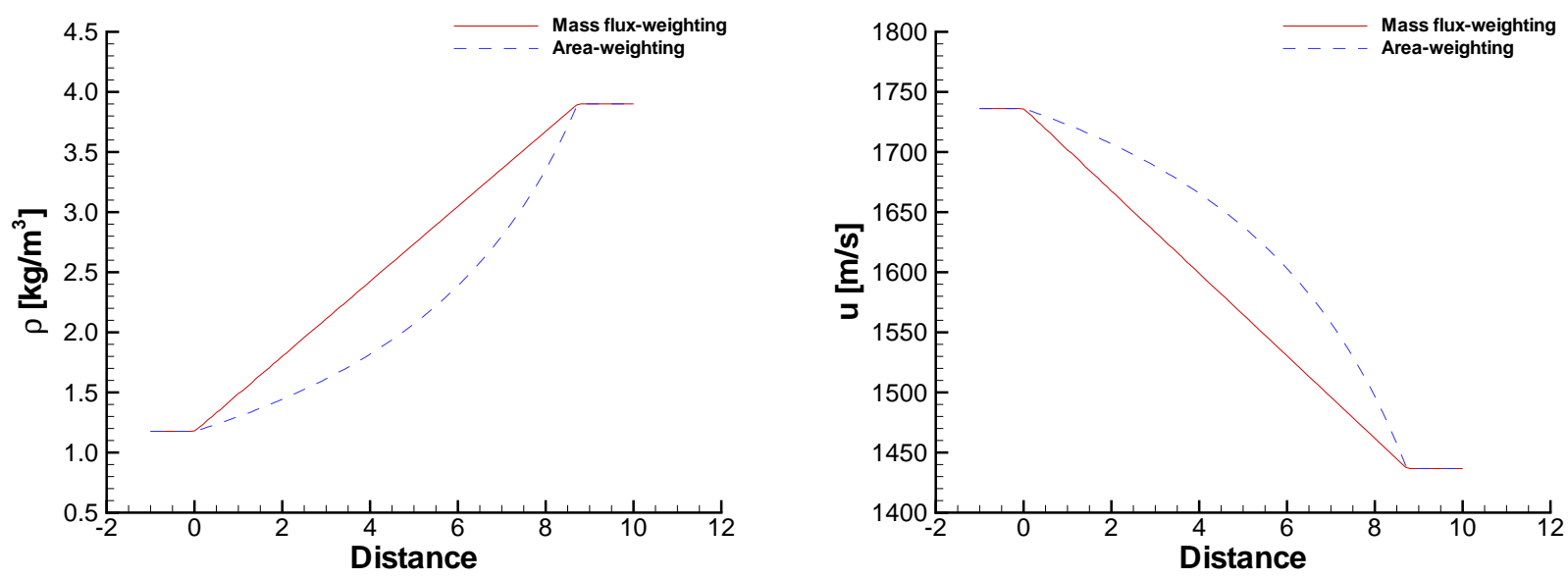

Figure 7: Static density and $x$-velocity distributions on grid 2 using weighted averages
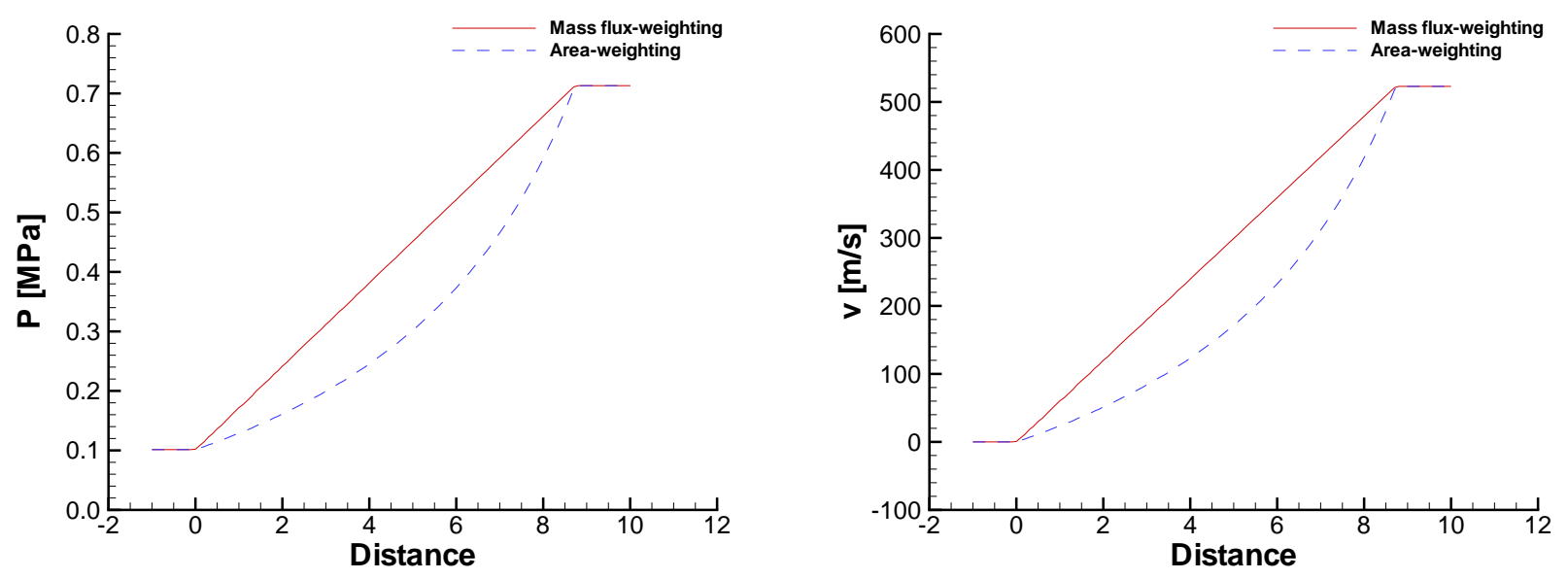

Figure 8: Static pressure and $y$-velocity distributions on grid 2 using weighted averages
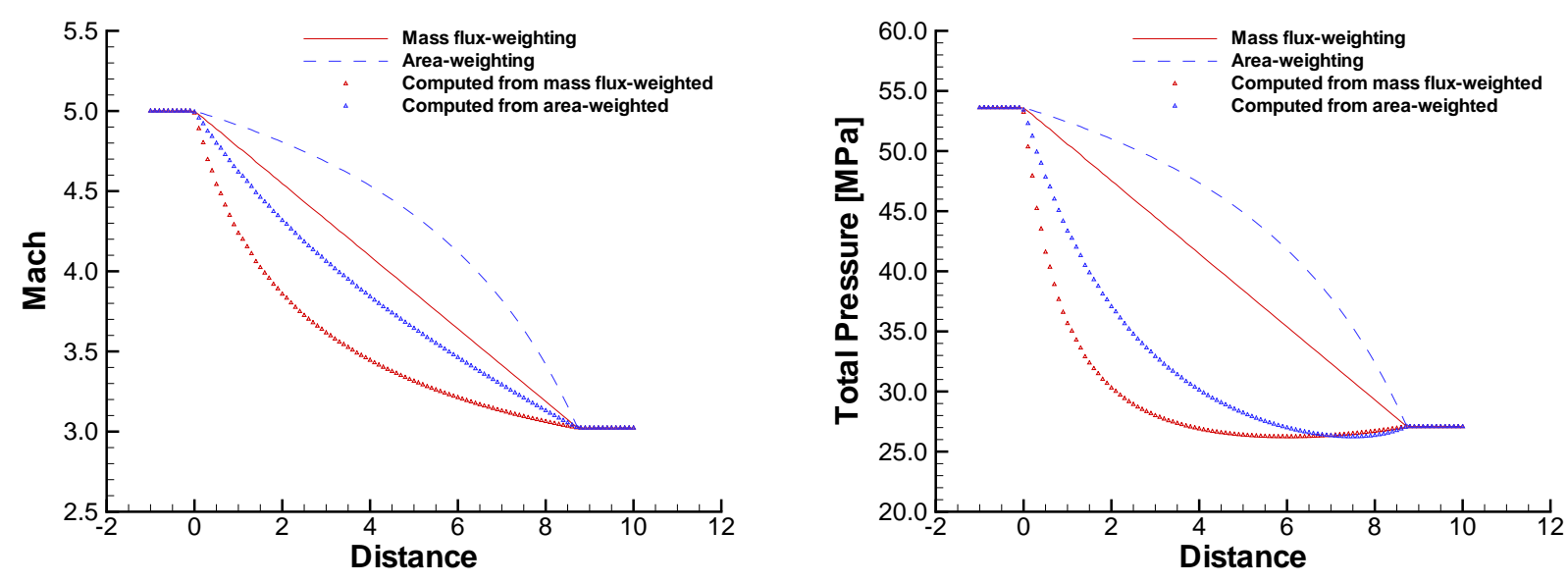

Figure 9: Mach number and total pressure distributions on grid 2 using weighted averages 

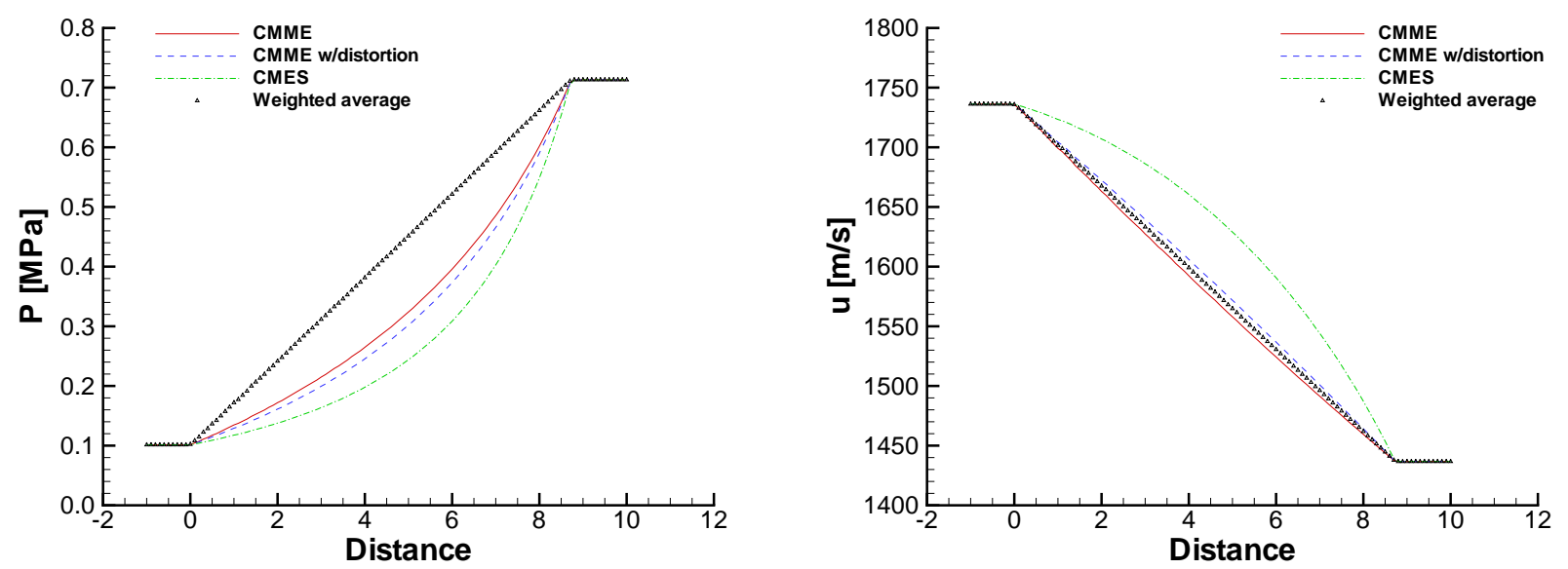

Figure 10: Static pressure and $x$-velocity distributions on grid 2 using conserved-flux approaches
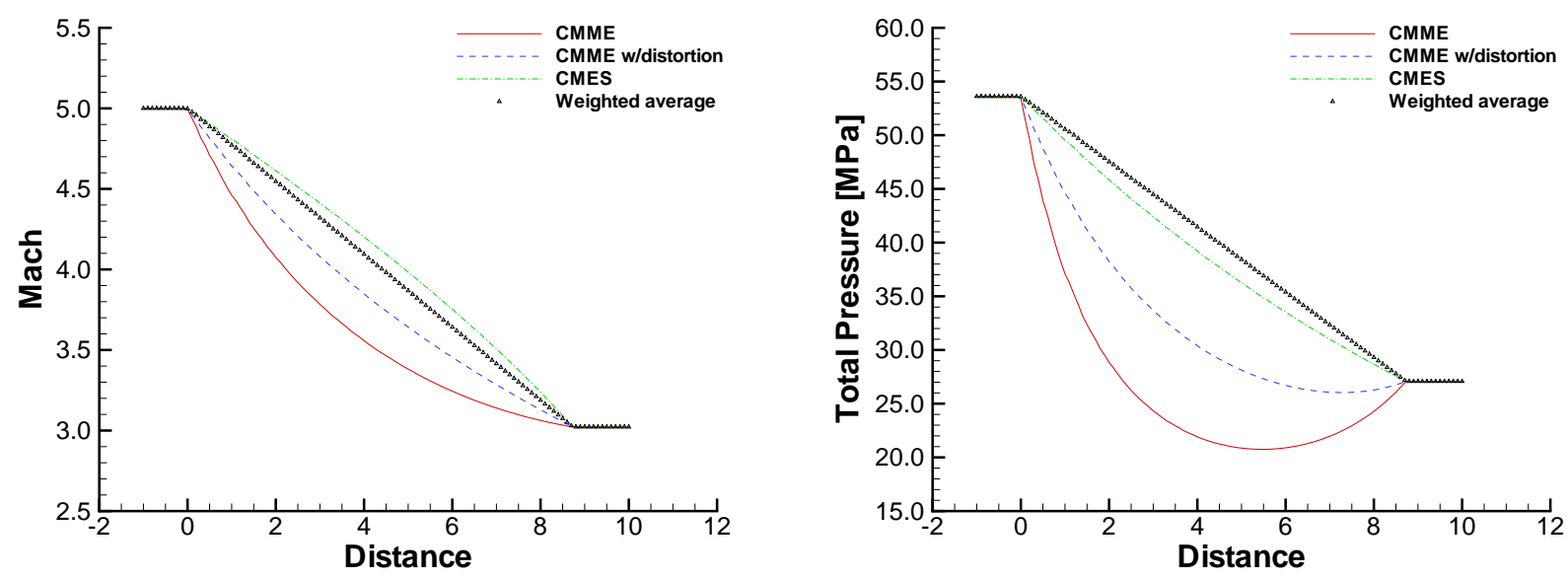

Figure 11: Mach number and total pressure distributions on grid 2 using conserved-flux approaches 


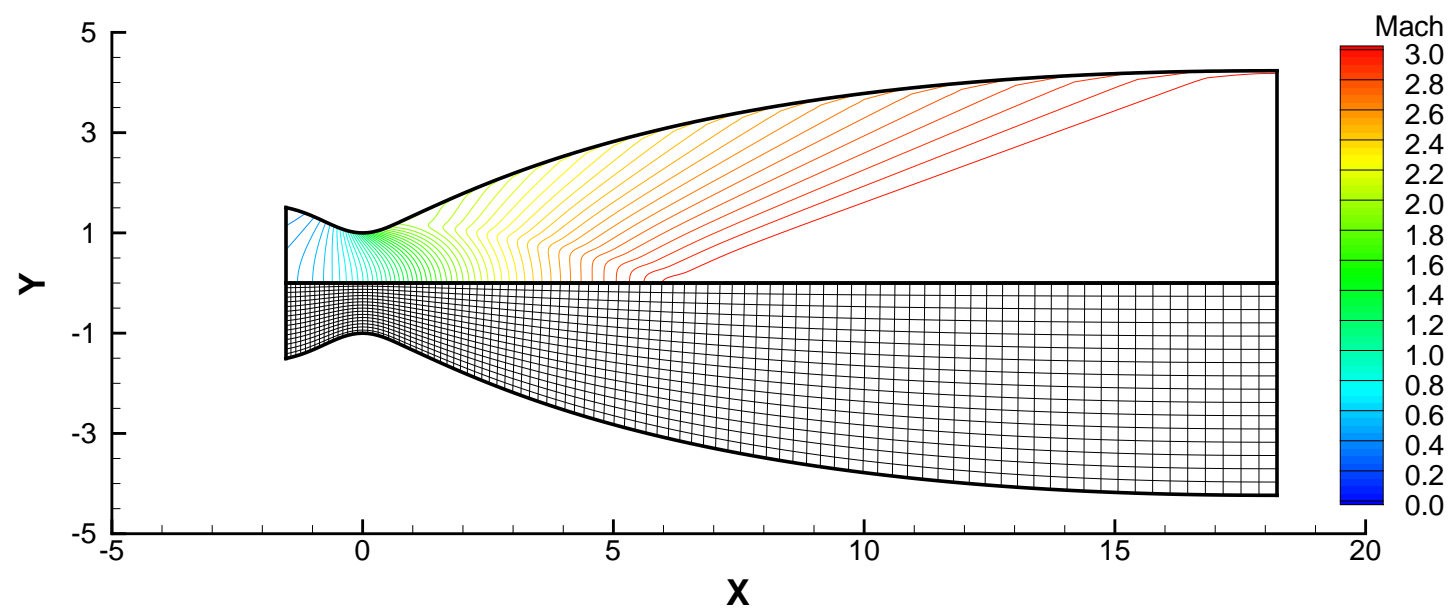

Figure 12: Grid (every fourth point removed for clarity) and Mach number contours for the nozzle
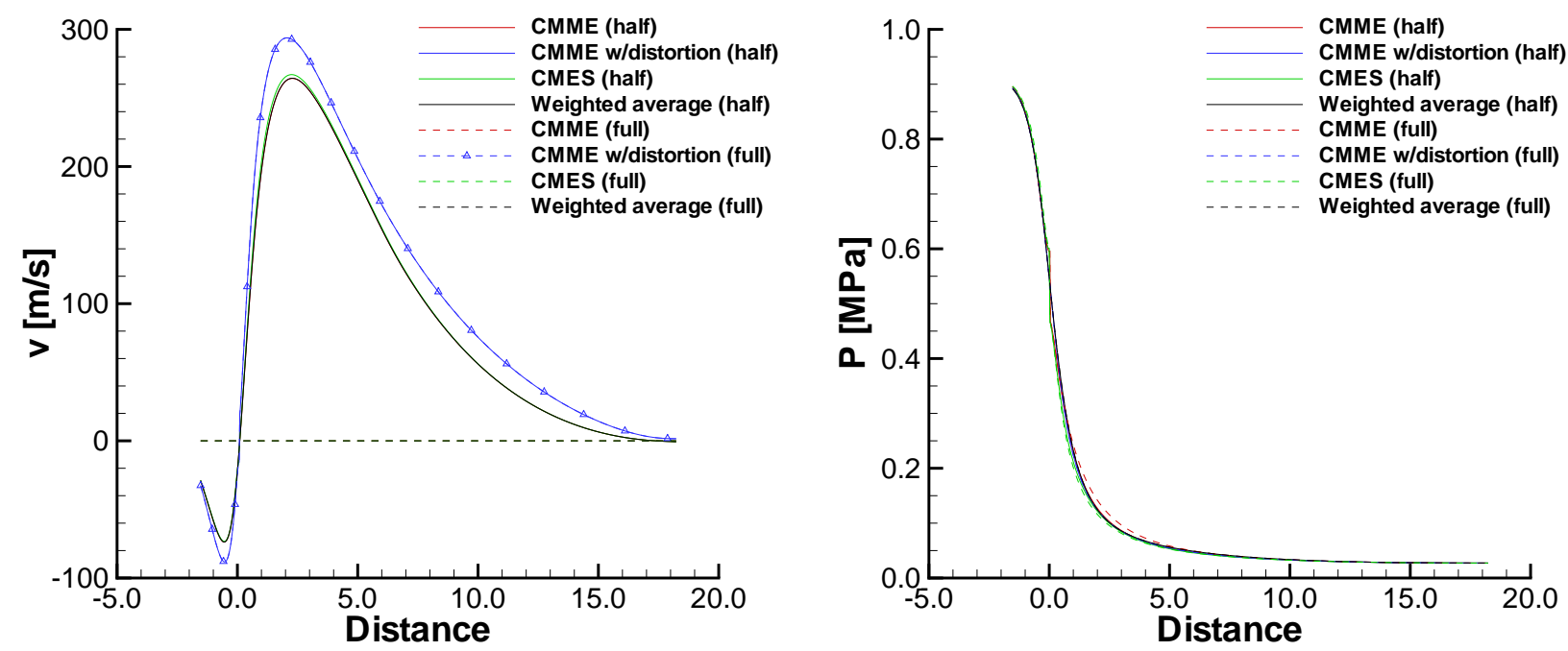

Figure 13: $y$-velocity and static pressure distributions for the half and full nozzle geometry
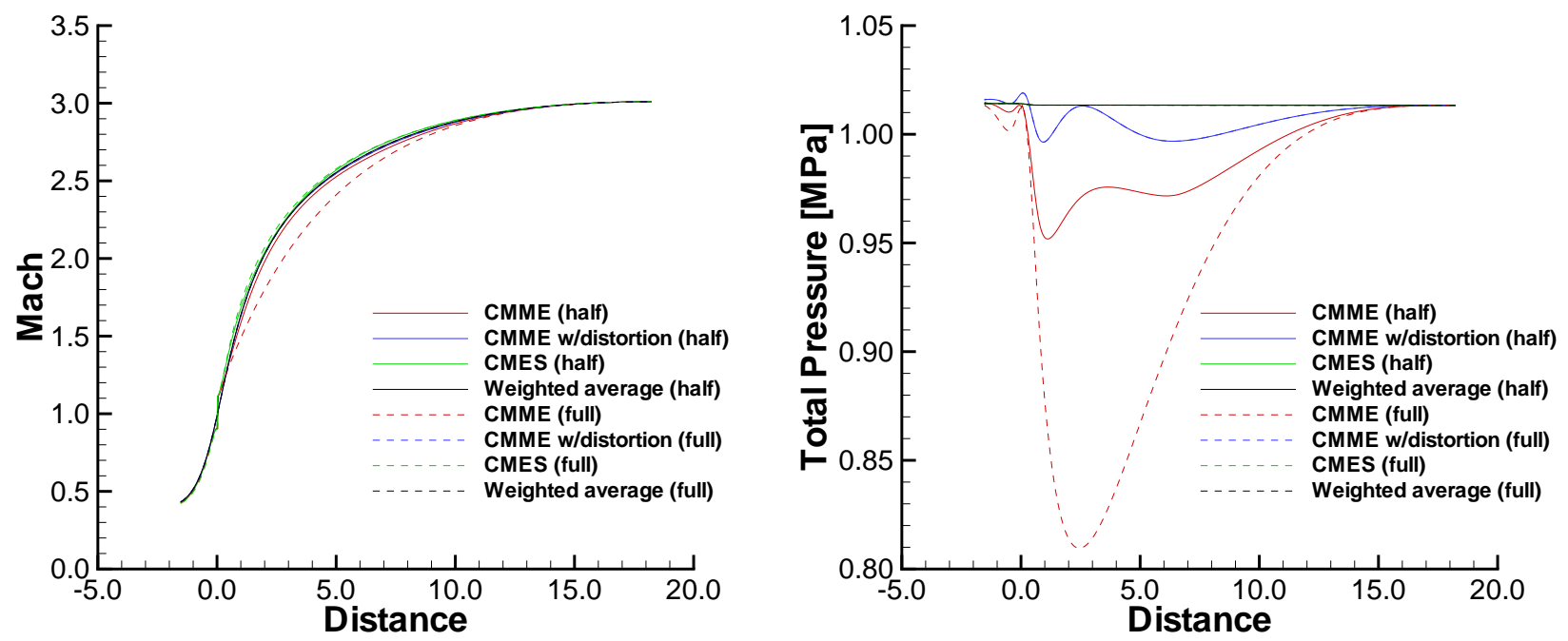

Figure 14: Mach number and total pressure distributions for the half and full nozzle geometry 

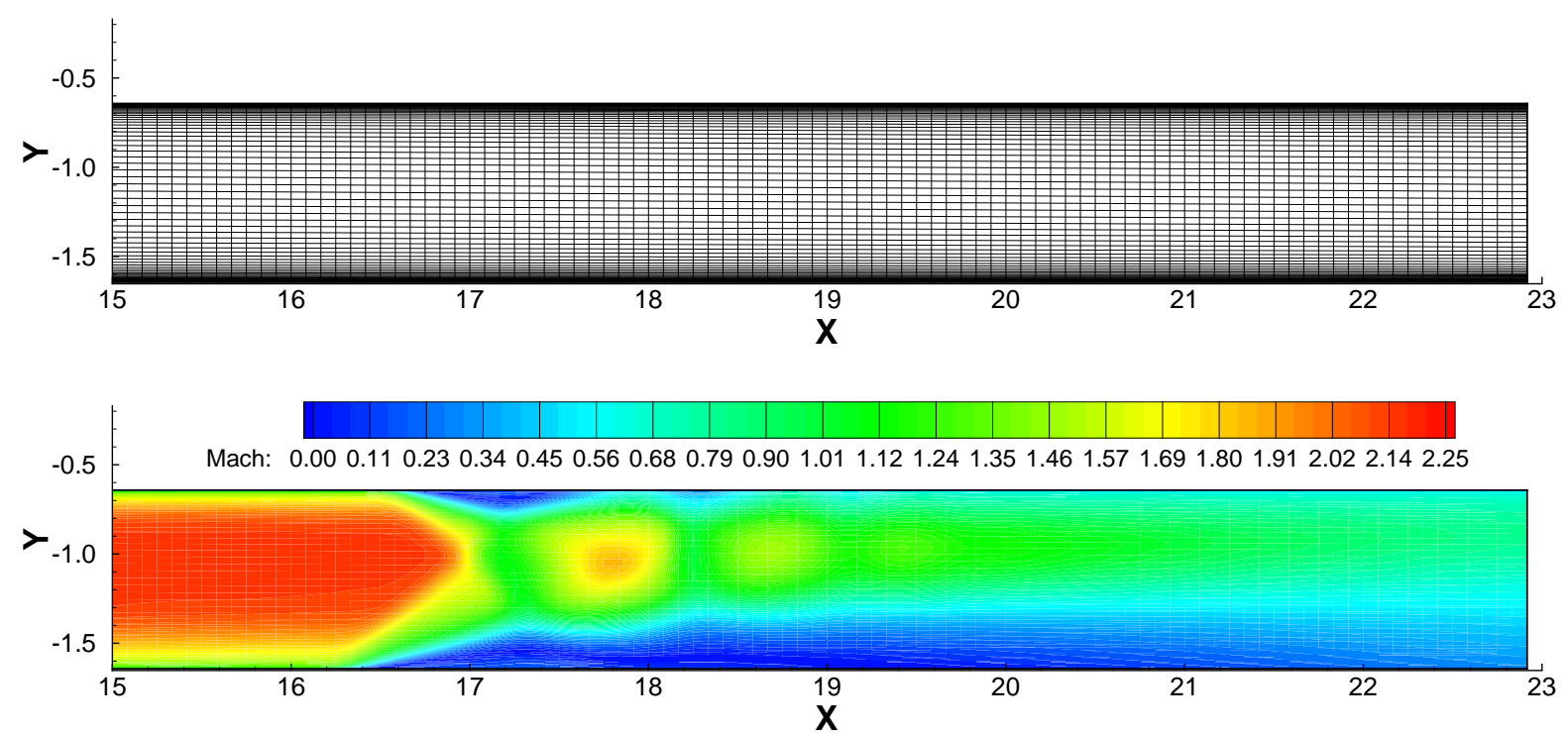

Figure 15: Grid and back-pressured isolator Mach contours
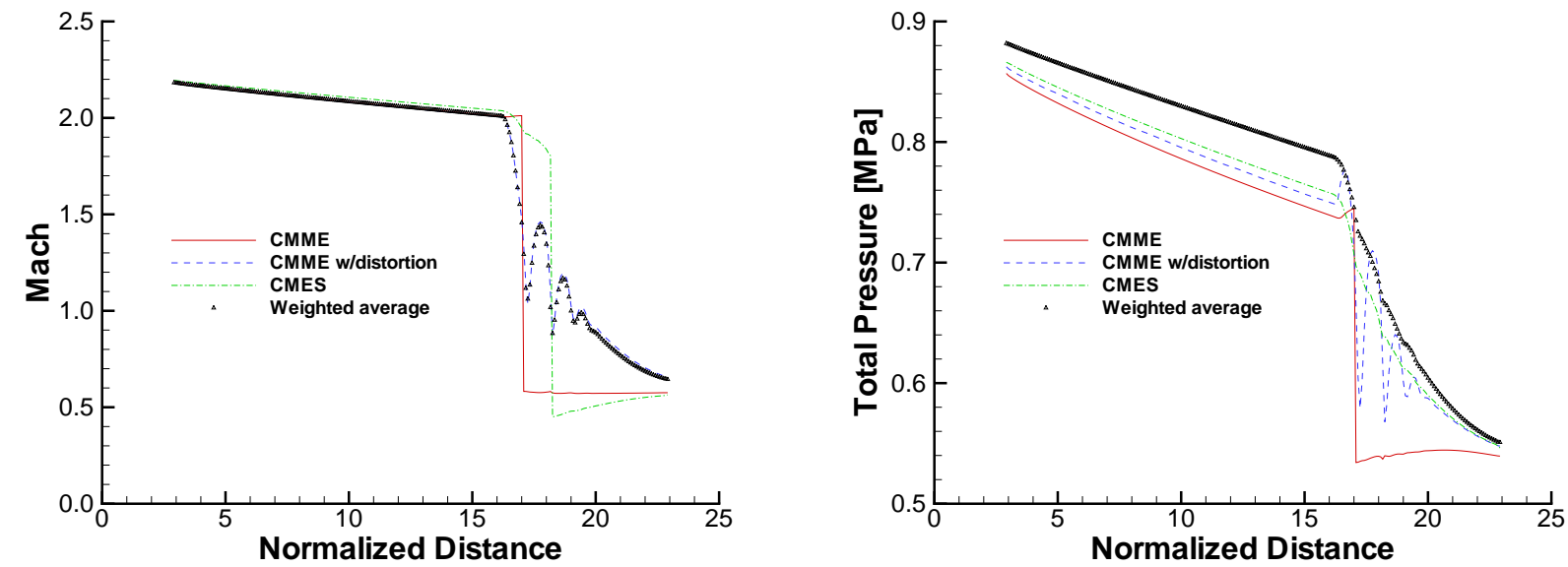

Figure 16: Mach number and total pressure distributions for the back-pressured isolator

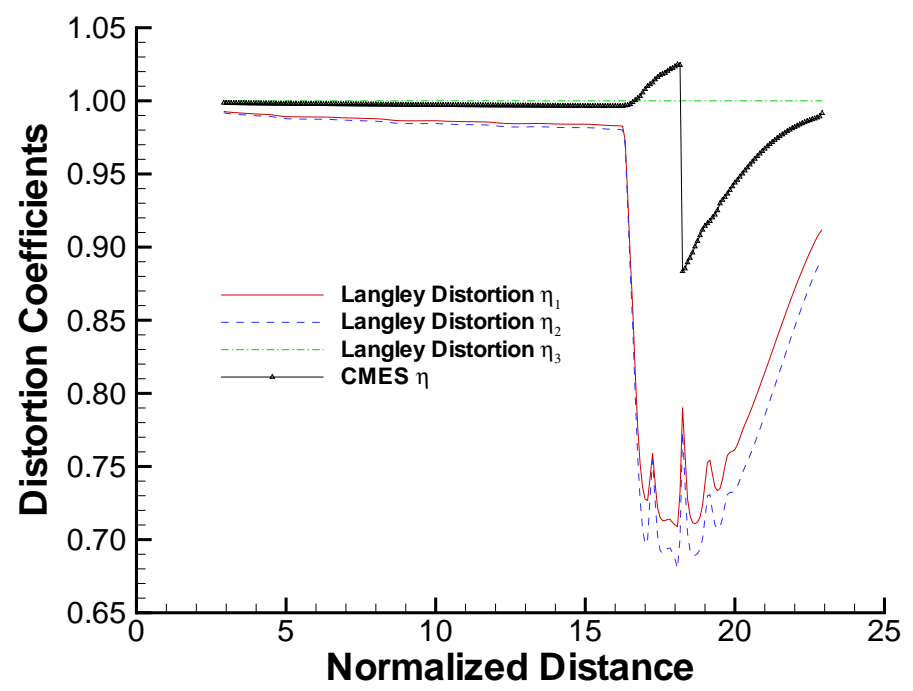

Figure 17: Distortion coefficients for the back-pressured isolator 